\title{
An Improved Model Predictive Control Method for Vehicle Lateral Control
}

\author{
Yunao $\mathrm{Li}^{1}$, Senchun $\mathrm{Chai}^{1}$, Runqi Chai ${ }^{2}$, Xiaopeng $\mathrm{Liu}^{3}$ \\ 1. School of Automation, Beijing Institute of Technology, Beijing 100081, P.R.China. \\ E-mail: bitliyunao@126.com; chaisc97@bit.edu.cn \\ 2. School of Aerospace, Transport and Manufacturing, Cranfield University, Bedfordshire MK43 0AL, United Kingdom \\ E-mail: 57545813@qq.com \\ 3. Institute of Telecommunication Satellite, China Academy of Space Technology, Beijing 100081, P. R. China \\ E-mail: liuxiaopeng2013@126.com
}

\begin{abstract}
This paper presents a lateral dynamic model based on control algorithm for the path tracking of autonomous vehicle. To improve the stability of the vehicle for high speed cases, an improved model predictive control (MPC) controller has been proposed in this paper. By combining the steady state response and MPC, the lateral motion of the autonomous vehicle can be controlled smoothly and the accuracy of path tracking can be guaranteed at a high speed. A number of simulation results obtained by using MATLAB are provided to validate this methodology.
\end{abstract}

Key Words: Autonomous vehicle, Lateral dynamics, Model predictive control, Steady state response, Stability

\section{Introduction}

Since the birth of autonomous vehicle, numerous algorithms have been proposed in order to control the motion of the vehicle [1]. According to references [2,3], it can be proved that the most of causes of vehicle accidents is coming from human beings, such as distraction [4] and wrong operation during the maneuver [5]. Therefore, several control solutions and advanced driving assistance systems were developed for autonomous car to avoid the happen of these problems and assist driver to control the cars more fluently [6].

In mechanical field, the majority of research focus on the longitudinal control action, i.e. the velocity in front direction [2]. However, the stability of vehicle not only depends on velocity displayed on panel, lateral control also should be considered to ensure the stability of car body and realtime pose adjustment, especially in high speed. Meanwhile, lateral control can implement trajectory tracking when the reference path is a known priori, which is generated by road condition or lead navigator [4].

Recently, model predictive control has been introduced in vehicle control problems [7, 8]. With the rapid increase of computing and sensing, the disadvantages of receding horizon can be overcome. MPC has the capability of solving the problems which could not be solved in real-time transport scenarios before.

Many researchers have studied autonomous driving system from different aspects, such as trajectory tracking and vehicle control. Saleh, Chevrel and Claveau presented an advanced driver assistance system for lane keeping derived from an $\mathrm{H} 2$ control problem [3]. Gu and Hu proposed a new path tracking scheme for a car-like mobile robot based on a backpropagation neural network, instead of traditional linear regression estimator [8]. Jazar introduced an auto-driver system in which the steering condition can be used to set the steer angles. The dynamic center of vehicle position can be controlled by adjusting the steer angles such that it coincides with the road curvature center [10].
In this paper, a simplified vehicle model is established based on classic bicycle scheme. Using the analysis of lateral dynamic, we introduce MPC algorithm to achieve control target on the vehicle model [9]. Different from traditional control strategy, such as proportional effect by error, we choose the steady state response as the controller inputs [10], which is more suitable for MPC. Besides, considering the constraints of input, a saturation function is designed to get better autonomous maneuvering of the controller.

\section{Vehicle Model}

\subsection{Vehicle Kinematics}

It is supposed that the model has no road gradient or bank angles and the vehicle has no load transfer and no rolling or pitching moment. Each couple of wheels lumps in two middle wheels to ignore the difference of steering angles. Moreover, the main assumption applied in this model is that the longitudinal speed is a constant. Only the front wheels angle can be controlled and the side slip angle transfers on a small scale [10].

With the consideration of the motions of vehicle chattering and the steering angle presenting nonlinear performance when entering large curve path, we select a parametric bicycle model to design MPC controller, illustrated in Fig. 1 $[10,11] . x, y, \theta$ mean the position and heading angle of the vehicle in global reference coordinates and $\beta, \omega$ mean two control variables in this model. The physical meaning of variables and parameters is shown in Table 1.

The discrete model is proposed by equation (1), where each variable value is shown using $\cdot_{i}$ in $i$-th time step and $\cdot_{i+1}$ at $(i+1)$-th time step, where $i \in(1, n), n \in N^{+}$.

$$
\left\{\begin{array}{l}
x_{i+1}=x_{i}+v_{i} \cdot \cos \left(\theta_{i}+\beta_{i}\right) \cdot \Delta t \\
y_{i+1}=y_{i}+v_{i} \cdot \sin \left(\theta_{i}+\beta_{i}\right) \cdot \Delta t \\
\theta_{i+1}=\theta_{i}+v_{i} \cdot \frac{\tan \left(\phi_{i}\right)}{L} \cdot \Delta t
\end{array}\right.
$$

The constraints of path curvature are given by equation (2). Equation (3) shows the nonholonomic constraint regarding different velocity components. 


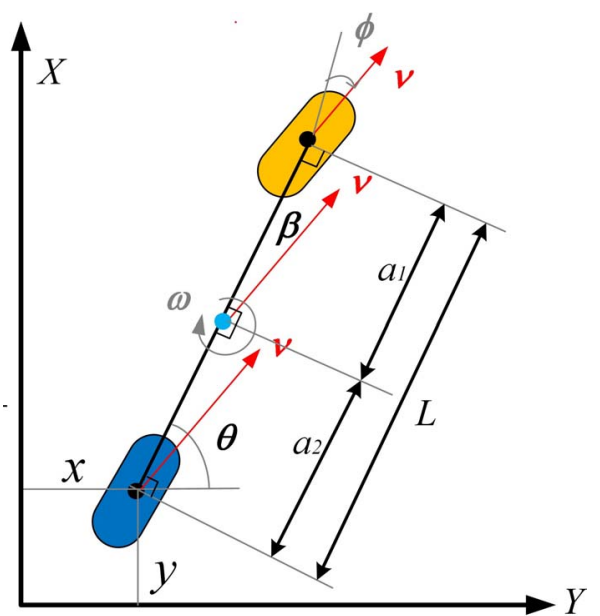

Fig. 1: Lateral dynamic of vehicle model

$$
\begin{gathered}
k_{\max }=\frac{1}{\rho_{\min }}=\frac{\tan \left(\phi_{\max }\right)}{L} \\
v_{x} \sin \theta-v_{y} \cos \theta=0
\end{gathered}
$$

Moreover, the sideslip angle of the vehicle $\beta$ is changing in a tiny scale, where $\beta$ can be approximated by equation (4).

$$
\beta \approx \tan \beta=\frac{v_{y}}{v_{x}}
$$

Table 1: Variables and Parameters

\begin{tabular}{l|l}
\hline \hline Symbol & Description \\
\hline$x_{i}$ & X position at $i$ instant (global reference coordinates) \\
\hline$y_{i}$ & Y position at $i$ instant(global reference coordinates) \\
\hline$\theta_{i}$ & Heading angle of vehicle at $i$ instant \\
\hline$v_{x}$ & Velocity component in longitudinal direction \\
\hline$v_{y}$ & Velocity component in lateral direction \\
\hline$\Delta t$ & Time interval \\
\hline$\phi$ & Steering angle \\
\hline$\beta$ & Side slip angle \\
\hline$\omega$ & Yaw rate \\
\hline$m$ & Mass of the vehicle \\
\hline$L$ & Wheelbase \\
\hline$k_{\max }$ & Path curvature upper bound \\
\hline$\phi_{\max }$ & Steering angle bound \\
\hline$a_{1}$ & Front distance from mass centre \\
\hline$a_{2}$ & Rear distance from mass centre \\
\hline$C_{\alpha f}, C_{\alpha r}$ & Tire coefficients \\
\hline$I_{z}$ & The moment of inertia matrix \\
\hline
\end{tabular}

\subsection{Lateral Dynamics}

To get higher tracking accuracy and vehicle stability performance, deducing the vehicle dynamics equations is required [12, 13]. Equations (5)- (7) describe the motion of the vehicle, where the linear tire model is used. Tire coefficients, $C_{\alpha f}$ and $C_{\alpha r}$ connect tire lateral forces and wheel slip. In longitudinal direction, fixed forward velocity can ensure that the model has an analytical solution. And most situations in high speed driving, such as lane change or lane keeping, are also considered at a fixed speed [10].

$$
\dot{v}_{x}=\frac{F_{x}}{m}+\omega v_{y}
$$

$$
\begin{aligned}
\dot{v}_{y}= & \frac{1}{m v_{x}}\left(-a_{1} C_{\alpha f}+a_{2} C_{\alpha r}\right) \omega \\
& -\frac{1}{m v_{x}}\left(C_{\alpha f}+C_{\alpha r}\right) v_{y}+\frac{1}{m} C_{\alpha f} \phi-\omega v_{x} \\
\dot{\omega}= & \frac{1}{I_{Z} v_{x}}\left(a_{1}^{2} C_{\alpha f}-a_{2}^{2} C_{\alpha r}\right) \omega \\
& -\frac{1}{I_{Z} v_{x}}\left(a_{1} C_{\alpha f}-a_{2} C_{\alpha r}\right) v_{y}+\frac{1}{I_{Z}} a_{1} C_{\alpha f} \phi
\end{aligned}
$$

Substituting equation (4) into (6), and then state-space equations are shown in (8) and (9). The equations represent the linear vehicle model can be determined by side slip and yaw rate.

$$
\dot{X}=A X+B \phi
$$

where,

$$
\begin{gathered}
X=(\beta, \omega)^{T} \\
A=\left(\begin{array}{cc}
-\frac{C_{\alpha f}+C_{\alpha r}}{m v_{x}} & \frac{-a_{1} C_{\alpha f}+a_{2} C_{\alpha r}}{m v_{x}^{2}}-1 \\
-\frac{a_{1} C_{\alpha f}-a_{2} C_{\alpha r}}{I_{Z}} & -\frac{a_{1}^{2} C_{\alpha f}-a_{2}^{2} C_{\alpha r}}{I_{Z} v_{x}}
\end{array}\right) \\
B=\left(\begin{array}{c}
\frac{C_{\alpha f}}{m v_{x}} \\
\frac{a_{1} C_{\alpha f}}{I_{Z}}
\end{array}\right) \\
Y=C X, \quad \text { where } \quad C=\left(\begin{array}{ll}
0 & 1
\end{array}\right)
\end{gathered}
$$

\section{Design of MPC Controller}

\subsection{Reference Path}

In this paper, we use B-spline curves to generate the reference paths to result continuous paths [14].

A $p$-th degree B-spline curve $c(u)$, can be defined by $n$ number control points, $P_{i}$, and $m$-number of knot vector, $\hat{u}$ , where $m=n+p+1$ and $p \in N^{+}$. The knot vector sequence $\hat{u}$ consists of $m$ non-decreasing real numbers, and $u$ is a normalized curve length variable.

In equation (10), $c(u)$ is the sum of each control point, $P_{i}$, and its basis function, $N_{i, p}(u)$. The expression of these functions is given by equation (11) and (12), which represent the first set of basis function and the higher degree basis functions from 2 to $p$ respectively.

$$
\begin{gathered}
c(u)=\sum_{i=0}^{n} N_{i, p}(u) P_{i} \\
N_{i, 0}(u)= \begin{cases}1 & u \in\left[\hat{u}_{i}, \hat{u}_{i+1}\right) \\
0 & \text { else }\end{cases} \\
N_{i, p}(u)=\frac{u-\hat{u}_{i}}{\hat{u}_{i+p}-\hat{u}_{i}} N_{i, p-1}(u)+ \\
\frac{\hat{u}_{i+p+1}-u}{\hat{u}_{i+p+1}-\hat{u}_{i+1}} N_{i+1, p-1}(u)
\end{gathered}
$$

Based on the B-spline methods, three designed reference paths are designed and generated, named Bend Turning(BT), Single Lane Change(SL) and Double Lane Change(DL), shown in Fig. 2- 4. 


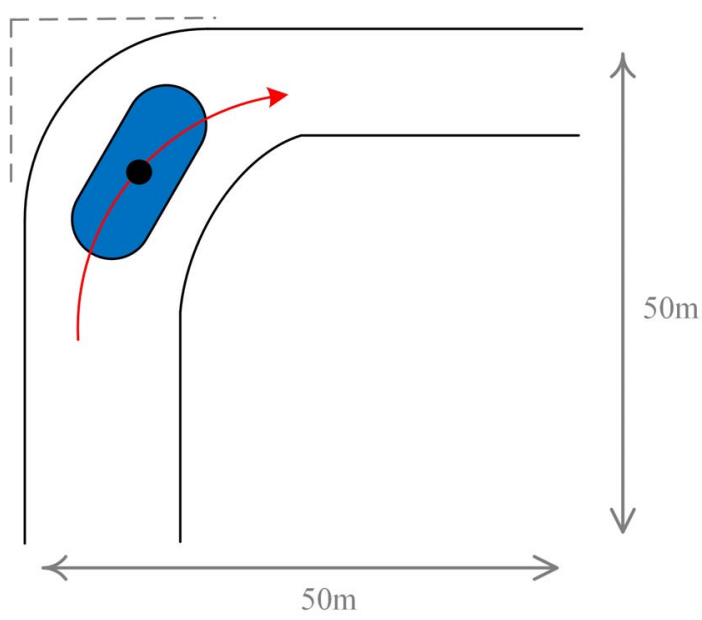

Fig. 2: Bend Turning (BT)

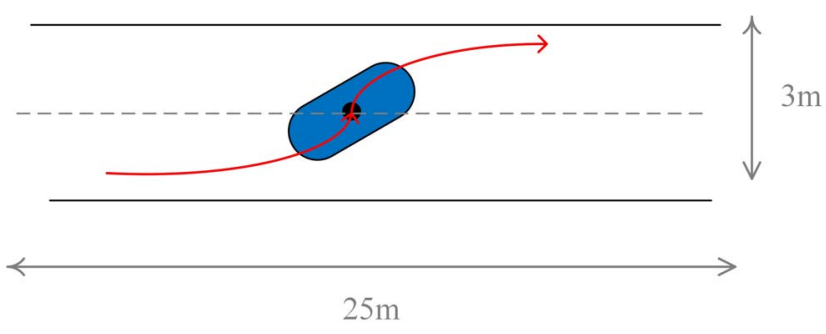

Fig. 3: Single Lane (SL)

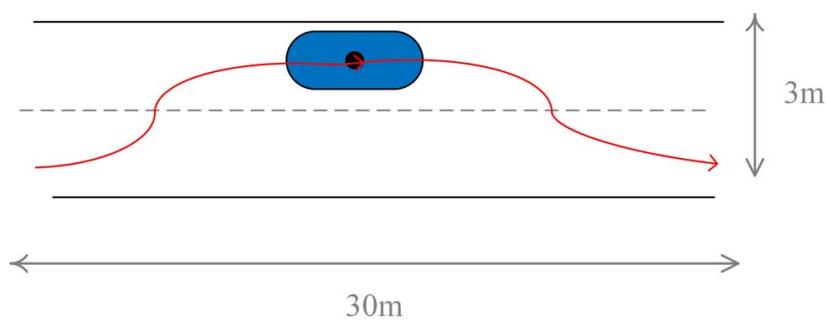

Fig. 4: Double Lane (DL)

\subsection{Control Scheme}

With the tracking trajectory created by B-spline method, the heading angle is calculated by linear parameter of reference curves at each instant, given in equation (13).

$$
\theta_{i}=\arctan \frac{y_{i}-y_{i-1}}{x_{i}-x_{i-1}}
$$

In traditional tracking control strategy, the error feedback is widely used, where the steering act is only controlled by the heading error. Given in equation (14), steering angle is in proportion to the heading error, where $k_{\theta}>0$ and $\theta^{\prime}$ means the actual value of $\theta$.

$$
\phi_{i}=k_{\theta}\left(\theta_{i+1}-\theta_{i}^{\prime}\right)
$$

To get better tracking performance of trajectory, an improved model predictive control is introduced to solve the problem of path-tracking. And the control scheme is illustrated in Fig. 5.

It can be seen that in the space-state equation (8), side slip and yaw rate are considered as state variables and optimal steering is the input of vehicle model. As a result, the task of MPC controller becomes offering an optimal value $\phi^{*}$ for vehicle at each instant.

According to reference [15], it is assumed that steady state steering is an approximated value of transient steering, which can be hold sufficiently close. So we can calculate the responses by equation (15) and (16), where C-coefficients and D-coefficients have been defined in reference [10].

$$
\begin{aligned}
& R_{\beta}=\frac{\beta_{i}}{\phi_{i}}=\frac{D_{\phi}\left(C_{\omega}-m v_{x}\right)-D_{\omega} C_{\phi}}{D_{\omega} C_{\beta}-C_{\omega} D_{\beta}+m v_{x} D_{\beta}} \\
& R_{\omega}=\frac{\omega_{i}}{\phi_{i}}=\frac{C_{\phi} D_{\beta}-C_{\beta} D_{\phi}}{D_{\omega} C_{\beta}-C_{\omega} D_{\beta}+m v_{x} D_{\beta}}
\end{aligned}
$$

where, $\quad C_{\phi}=C_{a f}, C_{\beta}=-\left(C_{a f}+C_{a r}\right)$,

$$
\begin{gathered}
D_{\phi}=a_{1} C_{a f}, D_{\beta}=-a_{1} C_{a f}+a_{2} C_{a r}, \\
C_{\omega}=\frac{-a_{1} C_{a f}+a_{2} C_{a r}}{v_{x}}, D_{\omega}=\frac{-a_{1}^{2} C_{a f}-a_{2}^{2} C_{a r}}{v_{x}}
\end{gathered}
$$

Next, we calculate the receding horizon results based on the lateral control model [16]. At each predictive horizon, the optimal steering change is calculated repeatedly by equation (17) and the optimal control for any instant is given in equation (18), for the prediction horizons, $N_{p}$, and control horizons, $N_{c}[9]$.

$$
\begin{gathered}
\Delta \phi_{i}^{*}=\left(\Phi^{T} \Phi+\bar{R}\right)^{-1} \Phi^{T}\left(\bar{R}_{s} r(i)-F x(i)\right) \\
\phi_{i+1}^{*}=\phi_{i}^{*}+\Delta \phi_{i}^{*}
\end{gathered}
$$

$\Phi$ and $F$ are derived using eqution (8) and (9), given in (19) and (20).

$$
\begin{gathered}
\Phi=\left(\begin{array}{ccccc}
C_{m} B_{m} & 0 & 0 & . . & 0 \\
C_{m} A_{m} B_{m} & C_{m} B_{m} & 0 & . . & 0 \\
. . & . . & . . & . . & . \\
\ddot{.} & . . & . . & . . & . \\
C_{m} A_{m}^{N_{p}-1} B_{m} & . . & . . & . . & C_{m} A_{m}^{N_{p}-N_{c}-1} B_{m}
\end{array}\right) \\
F=\left(\begin{array}{c}
C_{m} A_{m} \\
C_{m} A_{m}^{2} \\
C_{m} A_{m}^{3} \\
\cdots \\
C_{m} A_{m}^{N_{p}}
\end{array}\right)
\end{gathered}
$$

where $A_{m}, B_{m}$ and $C_{m}$ are the state matrix after discretization of model.

The controller tuning law is defined in equation (21), where $r_{w}$, as a tuning parameter, is considered to adjust controller performance from 0 (minimizing tracking error) to infinity (no control effort). And $\bar{R}_{s}$ is defined in equation (22).

$$
\begin{gathered}
\bar{R}=r_{\omega} I_{N_{c}, N_{c}} \\
\bar{R}_{s}=I_{N_{c}, 1}
\end{gathered}
$$

In this model, $x(i)$ is the current states $\left[X^{T}, Y\right]^{T}$ at $i$-th step and $r(i)$ is the desired steady state yaw set point [10]. 


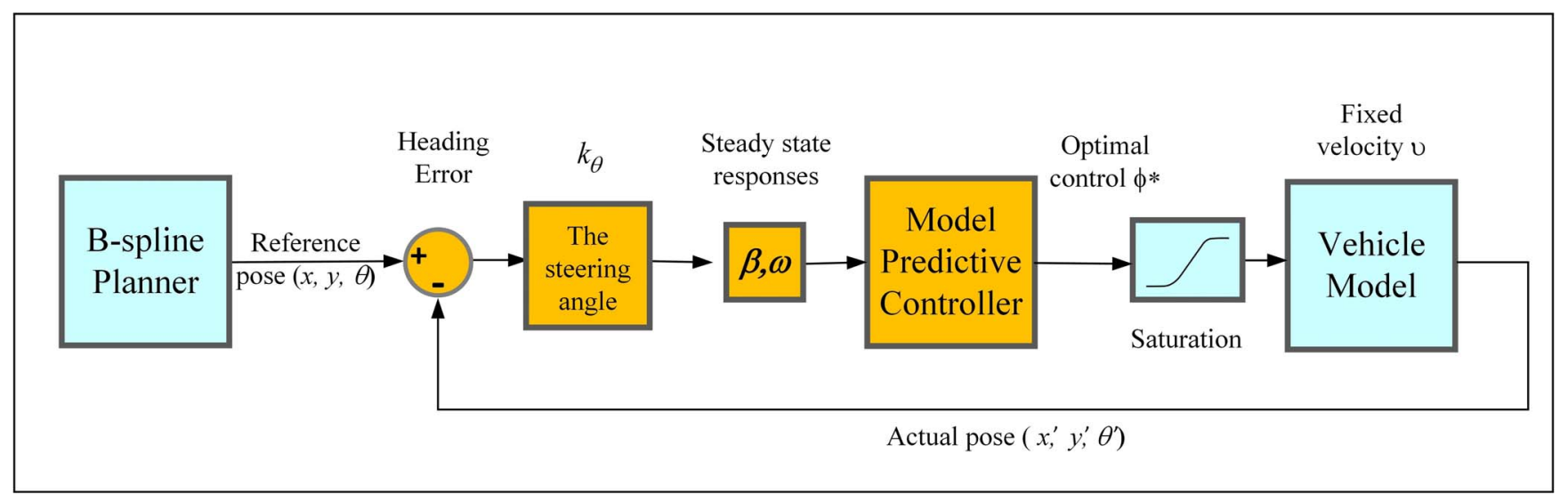

Fig. 5: Improved MPC control scheme

$r(i)$ is defined in equation (23). We can get $r(i)$ by equation (23).

$$
r(i)=R_{\omega} \cos \left(R_{\beta} \cdot \phi_{i}\right) \cdot \phi_{i}
$$

\subsection{Input Constraints}

In above-mentioned strategy, it is possible to have unreasonable inputs in the actual vehicle [17]. Considering Fig. 5 and equation (14)- (16), when the absolute value of heading error increasing over limitation, the side slip angle and yaw rate is following and the optimal control input, $\phi^{*}$ will output excessive control action. Therefore, to avoid this case, we introduce saturation limits as a compensation of the model at each sampling instant, which can restrain the oversize input bringing from excessive situation and measured errors.

Although majority of basic functions can be used to achieve the same end, a piecewise saturated function is given in equation (24) to smooth the bound of inputs, where the upper bound and lower bound is defined as ${ }^{-}$and :

$$
\bar{f}(x)= \begin{cases}f(x) & \underline{\varepsilon} \leq f(x) \leq \bar{\varepsilon} \\ \bar{\varepsilon}+\frac{f(x)-\bar{\varepsilon}}{1+\frac{f(x)-\bar{\varepsilon}}{\varepsilon}} & f(x)>\bar{\varepsilon} \\ \underline{\varepsilon}+\frac{f(x)-\underline{\varepsilon}}{1+\frac{f(x)-\underline{\varepsilon}}{\varepsilon}} & f(x)<\underline{\varepsilon}\end{cases}
$$

$\bar{\varepsilon}$ and $\underline{\varepsilon}$ are length of smooth transition, with $\bar{\varepsilon}=f(x)_{\max }-$ $\varepsilon$ and $\underline{\varepsilon}=f(x)_{\min }+\varepsilon$. $\varepsilon$ is a constant and $\varepsilon \in(0,1)$. An illustration of smooth saturation function is shown in Fig. 6 ( using $\varepsilon=0.5$, extremum $= \pm 10 \mathrm{deg}$ ) .

\section{Experiments results}

In this section, we discuss the stability and tracking performance shown in the results of simulation experiments. To validate the performance better, the experiments are executed in different environments and at relatively high speeds. The parameters of vehicle and path are shown in Table 2 and Table 3.

- Three designed environments: BT, SL and DL.

- Six reference speeds: 1, 2 ,5, $10,15,20$ [m/s]

- Control horizon and prediction horizons: $N_{c}=15$ steps, $N_{p}=50$ steps.

Because of the limited space, we select part of simulated results shown in Fig 7- 12. The side slip for BT, SL, DL at different speeds is shown in Fig 7- 9. And Fig 10 and Fig 11

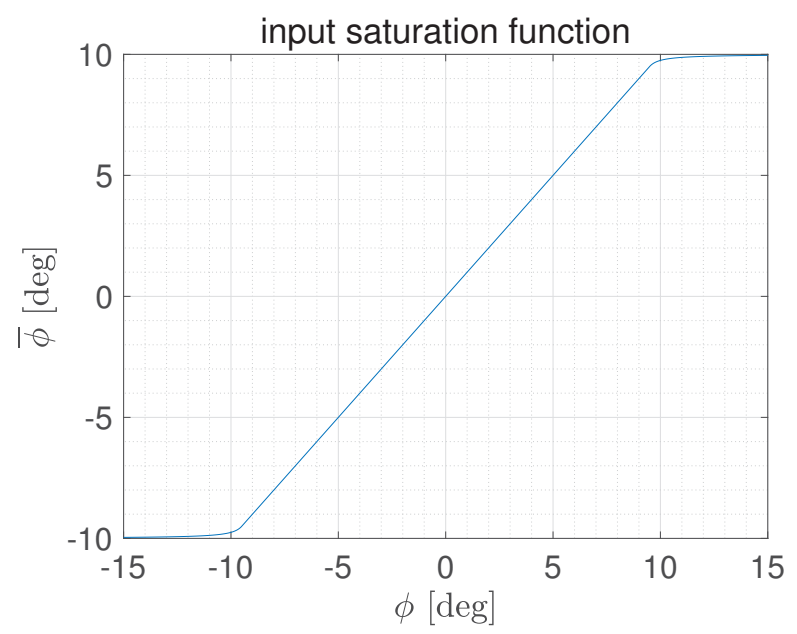

Fig. 6: The saturation function for side slip angle

show tracking performance of the vehicle in designed environment at different longitudinal speed. In BT environment, the reference and output yaw rate and the steering angle are shown in Fig 12.

Table 2: Vehicles Parameters

\begin{tabular}{l|l}
\hline \hline Parameter & \\
\hline $\mathrm{m}(\mathrm{Kg})$ & 1000 \\
\hline$I_{z}\left(\mathrm{Kg} / m^{2}\right)$ & 1650 \\
\hline $\mathrm{L}(\mathrm{m})$ & 2.6 \\
\hline$a_{1}(\mathrm{~m})$ & 1.0 \\
\hline$a_{2}(\mathrm{~m})$ & 1.6 \\
\hline$C_{\alpha f}(\mathrm{~N} / \mathrm{rad})$ & 3000 \\
\hline$C_{\alpha r}(\mathrm{~N} / \mathrm{rad})$ & 3000 \\
\hline$\phi_{\max }(\mathrm{deg})$ & 25 \\
\hline
\end{tabular}

Table 3: Path Parameters

\begin{tabular}{l|l|l|l}
\hline \hline Path & BT & SL & DL \\
\hline length $(\mathrm{m})$ & 90.11 & 30.24 & 125.43 \\
\hline Total heading change (degrees) & 90.0 & 22.62 & 25.11 \\
\hline
\end{tabular}

\subsection{Control Performance Analysis}

The control performance of vehicle lateral stability can be evaluated by measuring the side slip angle [18]. The value 
of side slip for BT, SL and DL cases using MPC at different speeds is illustrated in Fig. 7- 9 respectively.

From the simulated results, it is concluded that the MPC controller can steer the vehicle below the slide slip limitations for all experiments and speed. This is because the increasing error does not affect the controller directly, but is translated to steady state responses, which means the controller uses the steady state as input rather than relys on the heading error. Considering the saturation of inputs, we also design relative saturation function.

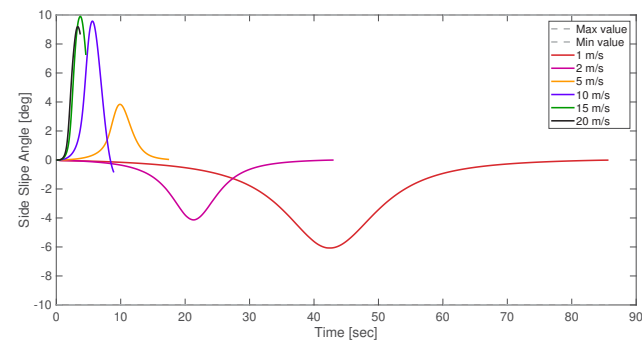

Fig. 7: Side slip angles for BT experiment at six speed

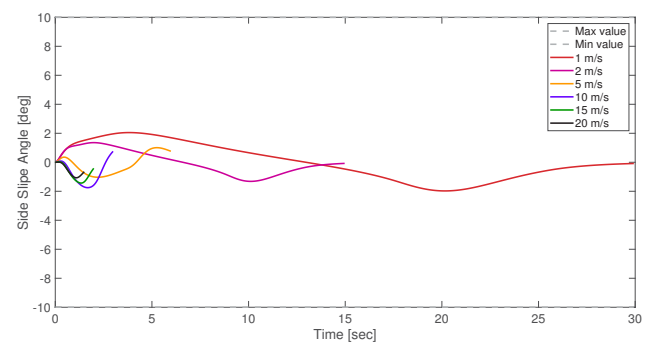

Fig. 8: Side slip angles for SL experiment at six speed

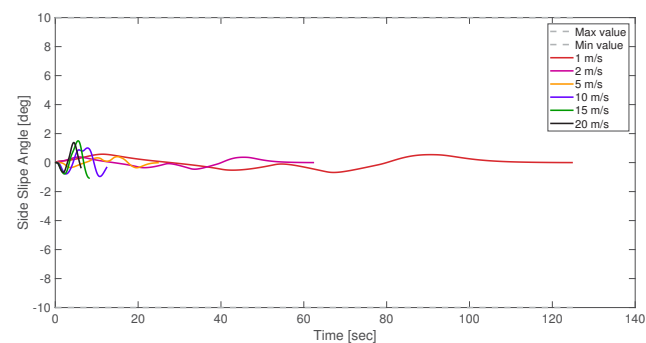

Fig. 9: Side slip angles for DL experiment at six speed

When the steering angles changing at higher speeds, it is unavoidable for side slip angle to sustain larger increases, which is also inferred in equation (15). The controller designed in this paper has the ability to maintain the slip angle in a small scale at high speed.

\subsection{Tracking Performance}

In this subsection, we discuss the tracking results in comparison to reference paths. The tracking paths in three environments at 20m/s are shown in Fig. 10. In SL and DL, the vehicle trajectory can follow the reference road timely, even in highest speed test case. In BT, because of the short time for adjustment and the larger steering angle in bend turning, the tracking performance is not as well as those in other environments.

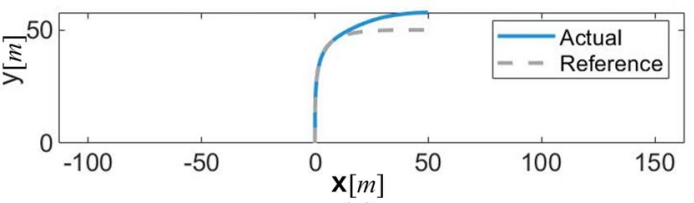

(a)

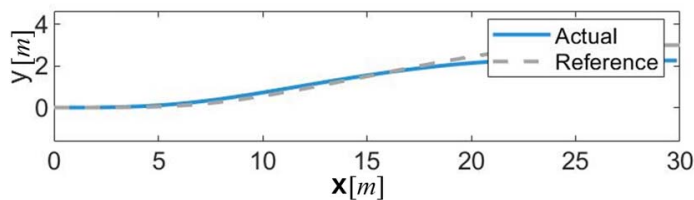

(b)

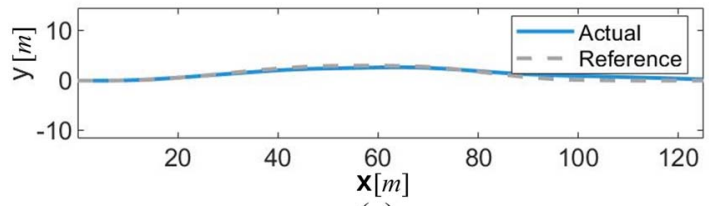

(c)

Fig. 10: Tracking performance in 3 experiments at 20m/s (a) BT (b) SL (c) DL

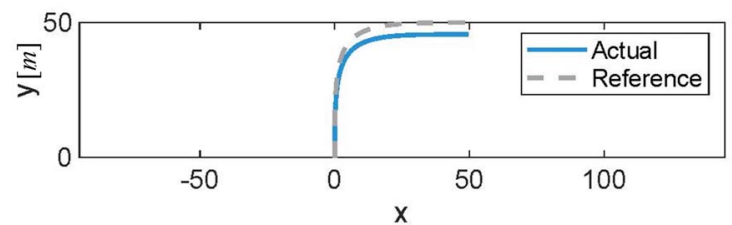

(a)

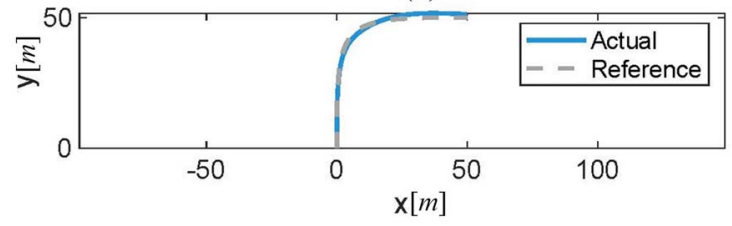

(b)

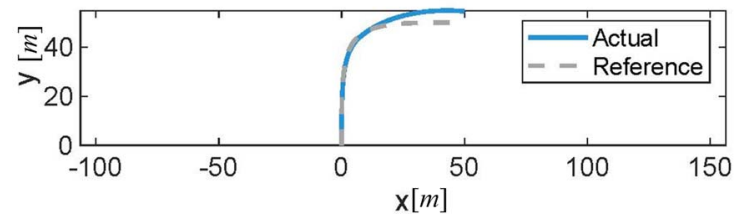

(c)

Fig. 11: Tracking performance in BT experiments at (a) $1 \mathrm{~m} / \mathrm{s}$ (b) $10 \mathrm{~m} / \mathrm{s}$ (c) $15 \mathrm{~m} / \mathrm{s}$

Furthermore in Fig. 11, it is illustrated that how the variables in tracking course changing at different speed in BT environment. In lower speed cases, the bend angle change is earlier than reference trajectory and in higher speed cases, the bend angle change is slower so the actual path is on the left.

And with the increase of speed, the delay of yaw rate is more obvious than the results shown in Fig. 12. This is because the action of vehicle can not follow the rapid change of steering angle timely. But the value of yaw rate and steering angle is maintained in a reasonable scale [19]. 


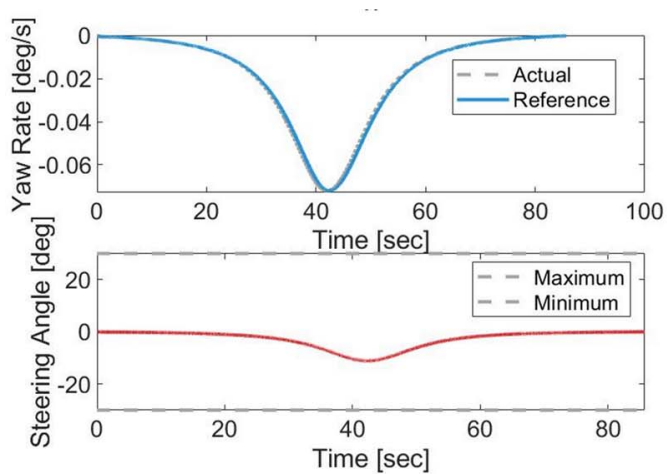

(a)

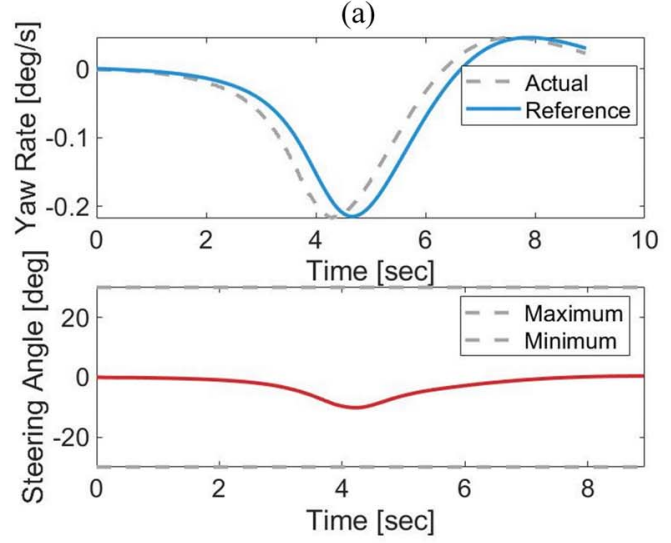

(b)
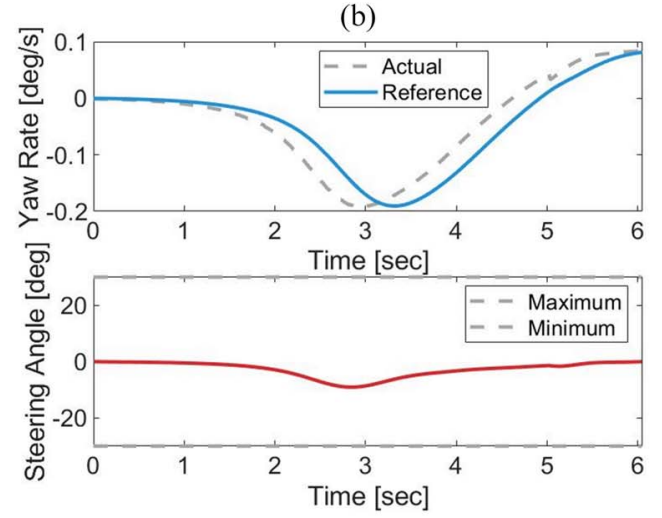

(c)

Fig. 12: The variables in tracking course for BT at (a) $1 \mathrm{~m} / \mathrm{s}$ (b) $10 \mathrm{~m} / \mathrm{s}(\mathrm{c}) 15 \mathrm{~m} / \mathrm{s}$

\section{Conclusion}

In this paper, a lateral dynamic model using path-tracking algorithm for autonomous vehicle is presented. An improved MPC controller has been proposed to improve the stability of the vehicle at high speed. The proposed design combines the steady state responses and model predictive control (MPC) method. According to the reported experimental results, it is observed that the lateral motions of the autonomous vehicle can be controlled smoothly and the path-tracking can be fulfilled at a high speed.

\section{References}

[1] M. Waldrop, Autonomous vehicles: No drivers required, $\mathrm{Na}$ ture, 518: 20-23, 2015.

[2] C. Laurgeau, Intelligent Vehicle Potential and Benefits, in Handbook of Intelligent Vehicles, A. Eskandarian Eds. London: Springer, 2012: 1537-1551.

[3] L. Saleh, P. Chevrel, F. Claveau, Shared Steering Control Be- tween a Driver and an Automation: Stability in the Presence of Driver Behavior Uncertainty, IEEE Trans. on Intelligent Transportation Systems, 14: 974-983, 2013.

[4] J. He, J. Mccarley, A. Kramer, et al, Lane Keeping Under Cognitive Load: Performance Changes and Mechanisms, Human Factors: The Journal of the Human Factors and Ergonomics Society, 56(2): 414-426, 2014.

[5] J. Lee, Fifty Years of Driving Safety Research, Human Factors: The Journal of the Human Factors and Ergonomics Society, 50(3): 521-528, 2008.

[6] K. Bengler, K. Dietmayer, B. Farber, et al, Three Decades of Driver Assistance Systems: Review and Future Perspectives, IEEE Intelligent Transportation Systems Magazine, 6(4): 6-22, 2014.

[7] D. Mayne, Model predictive control: Recent developments and future promise, Automatica, 50(12): 2967-2986, 2014.

[8] D. Gu, H. Hu, Neural predictive control for a car-like mobile robot, Robotics and Autonomous Systems, 39(2): 73-86, 2002.

[9] L. Wang, Model Predictive Control System Design and Implementation Using MATLAB. London: Springer-Verlag, 2009, chapter 2.

[10] R. Jazar, Vehicle Dynamics: Theory and Application. Boston: Springer-Verlag, 2008, chapter 3.

[11] G. Campion, G. Bastin, B. Dandrea-Novel, Structural properties and classification of kinematic and dynamic models of wheeled mobile robots, IEEE Transactions on Robotics and Automation, 12(1): 47-62, 1996.

[12] D. Dolgov, S. Thrun, Autonomous driving in semi-structured environments: Mapping and planning, in IEEE International Conference on Robotics and Automation, 2009: 3407-3414.

[13] L. Ma, J. Xue, K. Kawabata, et al, Efficient Sampling-Based Motion Planning for On-Road Autonomous Driving, IEEE Transactions on Intelligent Transportation Systems, 16(4): 1961-1976, 2015.

[14] C. Boor, On calculating with B-splines, Journal of Approximation Theory, 6(1): 50-62, 1972.

[15] H. Marzbani, A. Mohd, M. Simic, et al, Steady-state dynamic steering, in Proceedings of Smart Digital Futures, 2014: 493504.

[16] R. Sharp, H. Peng, Vehicle dynamics applications of optimal control theory, International Journal of Vehicle Mechanics and Mobility , 49(7): 1073-1111, 2011.

[17] B. Guerreiro, C. Silvestre, R. Cunha, et al, Trajectory Tracking Nonlinear Model Predictive Control for Autonomous Surface Craft, IEEE Transactions on Control Systems Technology, 22(6): 2160-2175, 2014.

[18] P. Tondel, T. Johansen Control allocation for yaw stabilization in automotive vehicles using multiparametric nonlinear programming, in American Control Conference, 2005: 453-458.

[19] Q. Cui, R. Ding, Z. Bing, et al, Path-tracking of an autonomous vehicle via model predictive control and nonlinear filtering, Proceedings of the Institution of Mechanical Engineers, Part D: Journal of Automobile Engineering, 232(9): 1237-1252, 2017. 
2020-09-09

\title{
An improved model predictive control method for vehicle lateral control
}

\author{
Li, Yunao
}

IEEE

Li Y, Chai S, Chai R, Liu X. (2020) An improved model predictive control method for vehicle lateral control. In: 2020 39th Chinese Control Conference (CCC), 27-29 July 2020, Shenyang, China https://doi.org/10.23919/CCC50068.2020.9188611

Downloaded from Cranfield Library Services E-Repository 